\title{
Structural and temporal heterogeneities on networks
}

\author{
Liubov Tupikina ${ }^{1 *}$ (D) and Denis S. Grebenkov ${ }^{2}$
}

\author{
* Correspondence: \\ liubov.tupikina@cri-paris.org \\ 1University Paris Descartes, Centre \\ of Research and Interdisciplinarity, \\ Paris, France \\ Full list of author information is \\ available at the end of the article
}

\begin{abstract}
A heterogeneous continuous time random walk is an analytical formalism for studying and modeling diffusion processes in heterogeneous structures on microscopic and macroscopic scales. In this paper we study both analytically and numerically the effects of structural and temporal heterogeneities onto the diffusive dynamics on different types of networks. For this purpose we investigate how the distribution of the first passage time is affected by the global topological network properties and heterogeneities in the distributions of the travel times. In particular, we analyze transport properties of random networks and define network measures based on the first-passage characteristics. The heterogeneous continuous time random walk framework, presented in the paper, has potential applications in biology, social and urban science, search of optimal transport properties, analysis of the effects of heterogeneities or bursts in transportation networks.
\end{abstract}

Keywords: Continuous time random walk, Stochastic processes on graphs, Diffusion, Complex networks, Heterogeneities, First passage time, Network measures

\section{Introduction}

Dynamical properties of random walks on networks are related to many vital questions, such as optimality and effciency of road systems, internet search strategy, functioning of metabolic networks (Barthelemy 2011; Montroll and Weiss 1965; Cohen et al. 2000). Dynamics on various types of random networks (graphs) (Sood and Redner 2005) have been extensively studied within the last decade and applied to describe epidemics and social processes (Brockmann et al. 2006). Many of large networks encountered in our everyday life have the so-called small-world property meaning that all nodes are closely connected (Agliari and Burioni 2009; Albert and Barabasi 2002; Julaiti et al. 2013). Moreover, it has been found that topological, geometric, and hydrological characteristics of a network system are directly linked to the characteristics of random walks on this network (Havlin and ben-Avraham 2002; Redner 2001), such as the first passage time (FPT), i.e. the time of the first arrival of the random walk to a target node (Bollt and ben-Avraham 2005). The first passage time characteristics are related to trapping problems, which play an important role in the control theory. Numerous problems on dynamical processes on networks (Hwang et al. 2012) include finding an appropriate placing of the trapping site in a network in order to obtain the desired trapping efficiency, search strategies etc. (Lambiotte et al. 2015). For homogeneous networks these problems have been studied extensively (Hudghes 1995; Maier

(c) The Author(s). 2019 Open Access This article is distributed under the terms of the Creative Commons Attribution 4.0 International License (http://creativecommons.org/licenses/by/4.0/), which permits unrestricted use, distribution, and reproduction in any medium, provided you give appropriate credit to the original author(s) and the source, provide a link to the Creative Commons license, and indicate if changes were made. 
and Brockmann 2017), for example, using the underlying backward equation approach (Bollt and ben-Avraham 2005). However, the analytical approaches, describing random walks on feature-rich (when nodes or links are endowed with some attributes (Monti and Boldi 2017)) heterogeneous networks, are lacking (Bollt and ben-Avraham 2005). It has been demonstrated that in continuous domains the mean FPT can differ from the most probable FPT (Godec and Metzler 2016; Grebenkov et al. 2018), in particular, moments of FPT do not fully reflect the transport efficiency. Moreover, since real-world networks are highly heterogeneous, reliable methods for analyzing transport properties on them are required (Valdano et al. 2018). To our knowledge, the complete description of the first passage quantities on various networks with structural or temporal heterogeneities has not been achieved. There is a particular reason for studying FPT properties on random networks. Some random networks have shown clear advantages over other network topologies (such as complete graphs) in terms of transport optimality and scaling efficiency, quantities which characterizes the change of the FPT density with the increase of network size in thermodynamic limit (Bollt and ben-Avraham 2005). Recently dynamics on random networks has been studied using the spectral and mean-field theories (Grabow et al. 2012; Mieghem 2011), which are not always applicable to heterogeneous networks.

In this article we consider Heterogeneous Continuous Time Random Walk (HCTRW) model (Grebenkov and Tupikina 2018) on several types of networks. The main motivation of the HCTRW model on networks is to study the influence of structural and temporal heterogeneities on diffusive dynamics. The HCTRW model is the extension of the conventional CTRW framework with additional heterogeneities, point-wise introduced to network sites or links (see "Model" subsection for more details). In the CTRW model, a random walker waits for random time between jumps driven from the same waiting time distribution $\psi(\mathrm{t})$ (Montroll and Weiss 1965). In the HCTRW model we relax the assumption of homogeneity of waiting time distributions between nodes: travel time distribution $\psi_{x x^{\prime}}(t)$ depends on both starting and ending points $\mathrm{x}$ and $x^{\prime}$. We investigate HCTRW on various graphs to see the interplay between dynamical random walk characteristics and structural properties of networks. The structure of the paper is the following. After presenting the HCTRW model in "Model" subsection, we investigate the first passage time of HCTRW on regular and irregular networks with structural and temporal heterogeneities in "Results" section. In "Discussions" and "Conclusions" sections we discuss the results and summarize the main findings.

\section{Model}

The Heterogeneous Continuous Time Random Walk is a model (Grebenkov and Tupikina 2018), in which a random walker moves on a graph $G$ in continuous time by jumping from one node to another with certain probabilities, set by a transition probabilities matrix $Q$ (further we refer to it as simply "transition matrix"). The element $Q_{x x^{\prime}}$ of the stochastic matrix $\mathrm{Q}$ is the probability of jumping from the node $\mathrm{x}$ to $x^{\prime}$ via link $e_{x x^{\prime}}$ and the travel time needed to move from $\mathrm{x}$ to $x^{\prime}$ is a random variable drawn from the probability density $\psi_{x x^{\prime}}(t)$. The transition probability and travel time distribution set the generalized transition matrix $\mathrm{Q}(\mathrm{t})$ with elements $Q_{x x^{\prime}}(t)=Q_{x x^{\prime}} \psi_{x x^{\prime}}(t)$. The 
heterogeneous travel times encode the structural and temporal heterogeneities, e.g. a trap $x_{h}$. The absorbing site $x_{a}$ of a network is a site, which has only the incoming but no outgoing links.

The propagator of HCTRW, $P_{x_{0} x}(\mathrm{t})$ (i.e., the probability density for a random walker started at $\mathrm{x}_{0}$ at time 0 to be at $\mathrm{x}$ at time $\mathrm{t}$ ), was obtained in (Grebenkov and Tupikina 2018) as

$$
\tilde{P}_{x_{0} x}(s)=\frac{1-\Sigma_{x^{\prime}} \tilde{Q}_{x x^{\prime}}(s)}{s}\left[(I-\tilde{Q}(s))^{-1}\right]_{x_{0} x}
$$

where $\tilde{P}_{x_{0} x}(s)$ (resp., $\tilde{Q}(s)$ ) is the Laplace transform of $P_{x_{0} x}(\mathrm{t})$ (resp., the generalized transition matrix $\mathrm{Q}(\mathrm{t})$ ). From Eq. (1) other important quantities of the process can be derived. In particular, the probability density $\rho_{x_{0}}(t)$ of the first passage time to a single absorbing node $\mathrm{x}_{\mathrm{a}}$ can be obtained using the renewal approach

$$
\tilde{p}_{x_{0}}(s)=\frac{\tilde{P}_{x_{0} x_{a}}(s)}{\tilde{P}_{x_{a} x_{a}}(s)} .
$$

When there are many absorbing nodes $x_{a}$, Eq. (2) is not applicable but there are other ways to compute the FPT density.

For each considered network (see below) we construct the transition matrix $\mathrm{Q}$ and the generalized matrix $\mathrm{Q}(\mathrm{t})$, given the set of the travel time distributions $\psi_{x x^{\prime}}(t)$ for each link $e_{x x^{\prime}}$. Then we perform the numerical computation of the inverse Laplace transform of $\tilde{\rho}_{x_{0}}(s)$ by Talbot algorithm (Talbot 1979) to get the FPT density $\rho_{x_{0}}(t)$ in time domain.

\section{Results}

We investigate the behavior of the FPT probability density on several types of networks. In each of these networks, we select an absorbing node $x_{a}$ and calculate the probability density $\rho_{x_{0}}(t)$ of the FPT to this node from a prescribed starting point $\mathrm{x}_{0}$. In this article we focus on the effects of heterogeneity by introducing a trap node $x_{h}$, at which the travel time distribution is different from the remaining nodes. Although the HCTRW model allows for arbitrary travel time distributions, we restrict our analysis to the simplest case of the exponential distribution. In this case $\psi_{x x^{\prime}}(t)=e^{-t / \tau} / \tau$, where $\tau$ is the mean travel time and $\tilde{\psi}_{x x^{\prime}}(s)=1 /(s \tau+1)$ is the Laplace transform of $\psi_{x x^{\prime}}(t)$. When a random walk arrives at the trap node, it is kept there for much longer times than $\tau$. This mechanism could be realized by using the exponential distribution with a much larger mean travel time. However, to highlight the effect of the trap node (heterogeneity) $x_{h}$, we will consider the distribution with infinite mean travel time, typically of the form $\tilde{\psi}_{x_{h} x^{\prime}}(s)=1 /\left(1+s^{a} \tau^{a}\right)$, with an exponent $\alpha$ between 0 and 1 . We will investigate how the presence of such a trap node and of eventual links avoiding this trap affect the FPT density.

We start with the HCTRW on regular structures, such as one-dimensional lattice chain and finite fractals ("HCTRW on regular graphs" subsection), then we study FPT properties on random networks ("HCTRW on random networks" subsection). In "Analytical insights onto the FPT density" subsection we give some analytical insights 
onto the FPT density on networks. Finally, we finish with a real-world network example ("Applications to real-world networks" subsection).

\section{HCTRW on regular graphs}

We use the following setup of the HCTRW model on regular loopless graphs. After setting up the trap $x_{h}$ and locating the absorbing node $x_{a}$ in a given graph we add an avoiding link $e_{x_{h}^{\prime} x^{\prime \prime}} ;$ e.g. in the case of the chain, the nodes $x_{h}^{\prime}$ and $x_{h}^{\prime \prime}$ are around the trap node $x_{h}$. Then we compute FPT densities for the cases with and without the avoiding link.

First, we consider the HCTRW on a one-dimensional chain with one absorbing node at $\mathrm{x}_{\mathrm{a}}=1$, one reflecting node $\mathrm{x}_{\mathrm{r}}=100$ and one trap node $\mathrm{x}_{\mathrm{h}}$, which is located either to the left or to the right from $x_{0}$. The corresponding FPT densities (see Fig. 1) show the same long-time behavior $t^{-t-a}$, discussed in (Grebenkov and Tupikina 2018). This result can be understood from the fact that a random walk on a one-dimensional chain with or without avoiding link will eventually get into a trap node even though an avoiding link potentially is allowing for a random walker to overjump over the trap.

Second, we consider a generalized Vicsek fractal, $\mathrm{Gv}_{g f}$, as an example of regular loopless structures, which resembles the dendrimers construction (Blumen et al. 2004; Liu et al. 2015). The fractal $\mathrm{Gv}_{g f}$ is constructed iteratively in a deterministic way by going from generation $\mathrm{g}$ to generation $g+1$ with coordination number $f$. A Vicsek fractal graph for $f=3$ and $g=3$ is shown in Fig. 2 (top). At the same time, it is known that the spectral properties of graph Laplacians are reflected in dynamical properties of random walks on them. In the case of the Vicsek fractal, the graph Laplacian obeys the simple scaling, determined by the spectral dimension of the Vicsek fractal $d_{s}=\frac{\ln (f+1)}{\ln (3 f+3)} \approx 0.557$.

We use the HCTRW setup, where all travel time distributions are $\tilde{\psi}_{x x^{\prime}}(s)=1 /(1+s \tau)$, except for the trap node $x_{h}$, at which $\tilde{\psi}_{x_{h} x^{\prime}}(s)=1 /\left(1+s^{a} \tau^{a}\right)$ with $\alpha=0.5$. We compute the FPT density on a Vicsek fractal for two positions of the trap: when the trap $x_{h}$ is

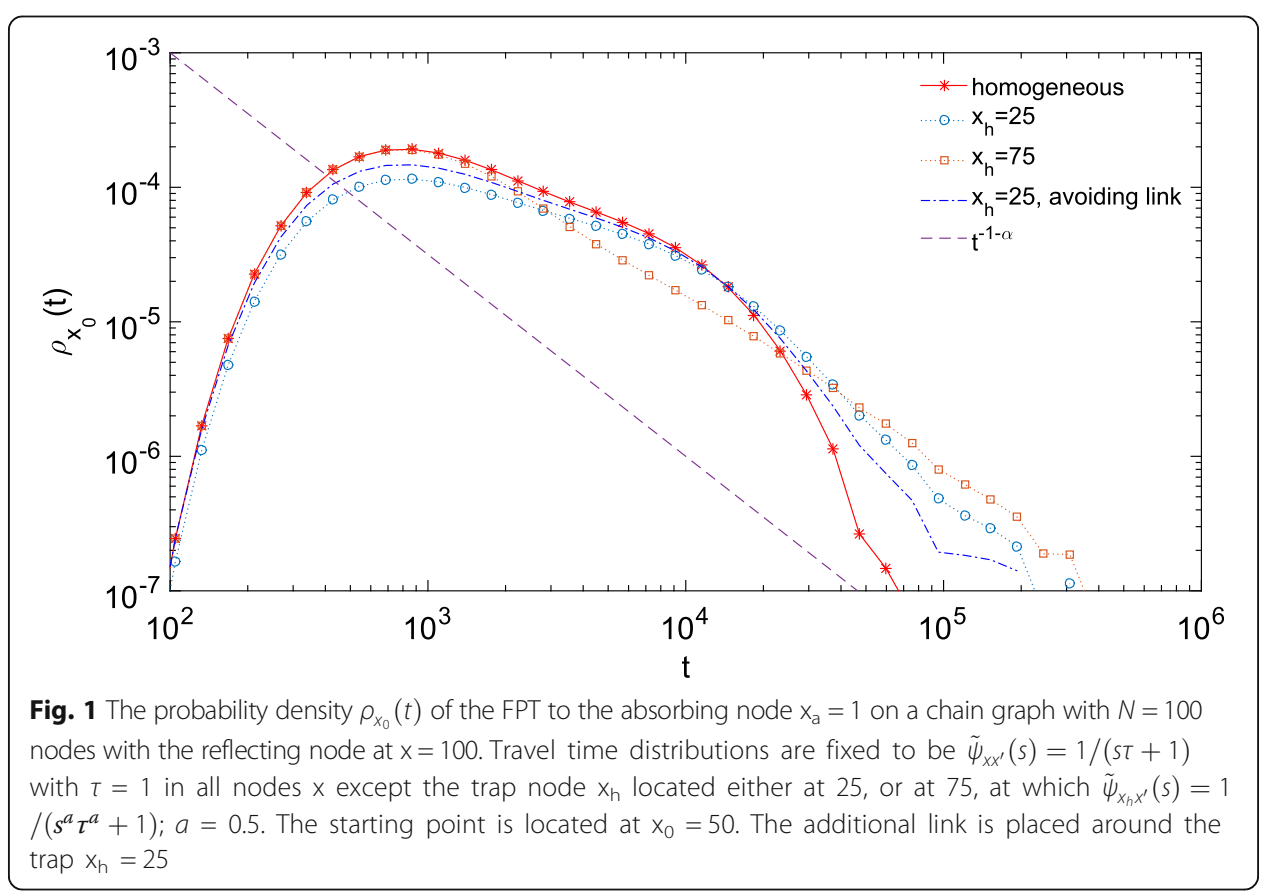




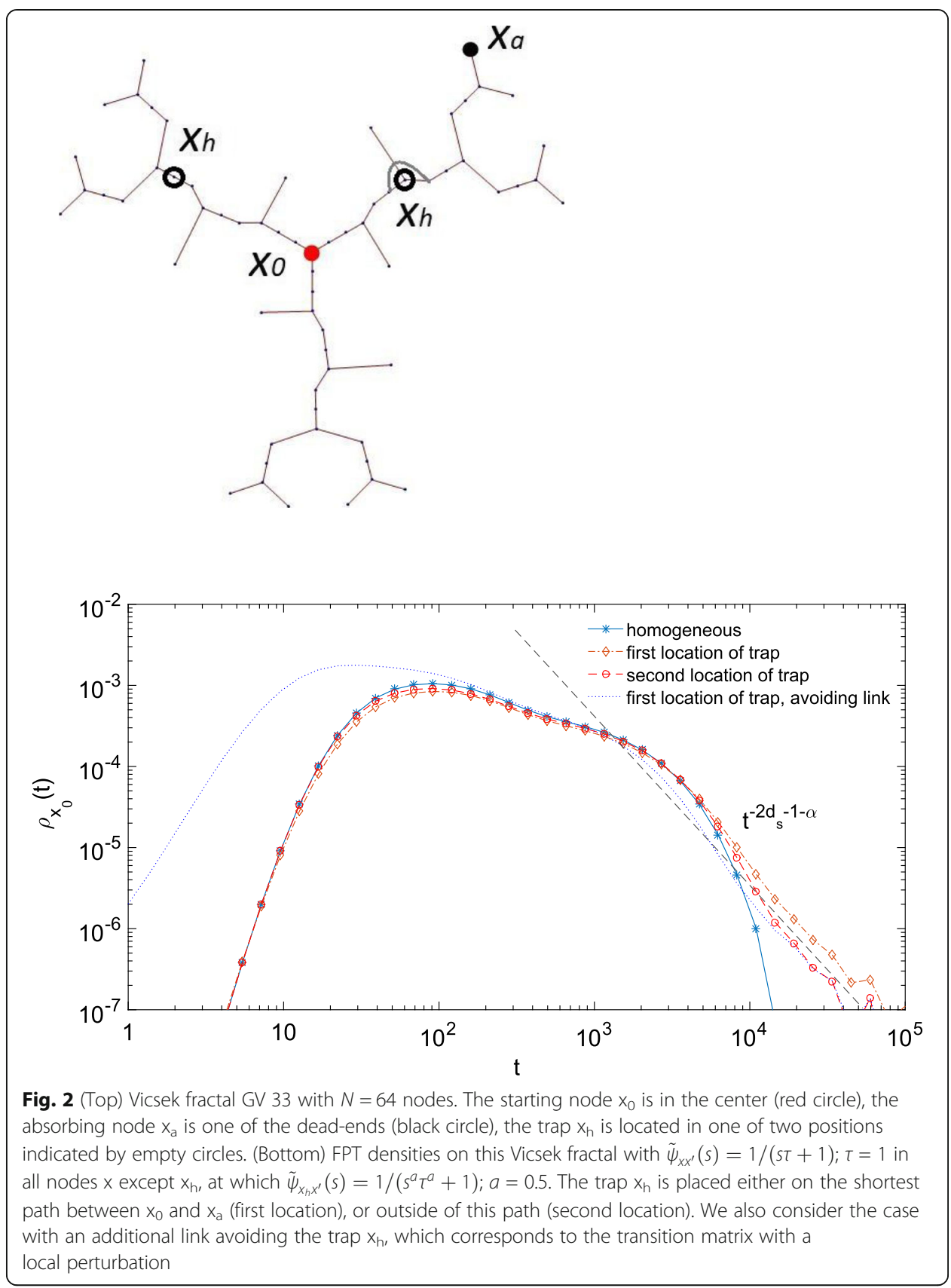

placed on the shortest path between $x_{0}$ and $x_{a}$, or outside of this path, shown on Fig. 2 (top). As we observe from Fig. 2 (bottom) the short-time regime of the FPT density with avoiding link differs from that without avoiding link, whereas these cases give the same power-law scaling $t^{-2 d_{s}-1-a}$ in the long-time regime, where $d_{s}$ is the spectral dimension of the Vicsek fractal.

\section{HCTRW on random networks}

Here we consider two classes of random networks: Scale-Free (SF) and Watts-Strogatz (WS) model. We construct SF networks using the preferential attachment 
Barabasi-Albert model $G\left(N, m, m_{0}\right)$, where $N$ is the number of nodes, $m$ is the number of initially placed nodes in a network and $m_{0}$ is the number of nodes, a newly added node is connected to (Albert and Barabasi 2002). The preferential attachment mechanism drives the network degree distribution to obey a power law decay with the exponent $\gamma \approx 3$ (von der Hofstadt 2017). Both, SF networks with and without loops show the small-world property.

The small-world Watts-Strogatz network model is denoted by $G(k, \beta)$, where $k$ is the average network degree and $\beta$ is the rewiring parameter (Watts and Strogatz 1998). WS model is constructed from a regular ring lattice, a graph where each node is connected to $k$ neighbors, $k=2$ on each side, where each link is then rewired with the probability $\beta$.

We numerically compute the FPT density on these random networks. We begin with demonstrating the FPT densities on one random realization of SF network, while we also checked that results are qualitatively the same when considering an ensemble of random networks.

First, we investigate the influence of a single trap placed in different communities of SF networks. The network communities structure, where each community is a group of nodes, which are interlinked with each other more than with other nodes, was extensively studied in the last decades (Barthelemy 2011; Schaub et al. 2017). Here we answer a more simple question: how a random walk can "see" the places of the trap in different areas of random networks. We choose non-intersecting communities in SF network, which are connected through the only edge to each other. Then we choose the absorbing node $x_{a}$ on this edge, as shown in Fig. 3 (top) and consider two cases by placing a single trap in two different communities with respect to the node $x_{a}$ : (a) trap is placed on a path (maybe not the shortest) between $x_{0}$ and $x_{a}$, denoted as $x_{0}<x_{h}<x_{a}$, hence a random walk cannot avoid getting into the trap $x_{h}$; (b) trap is placed in another community in respect to $x_{0}$ node, such that a random walk is able to reach $x_{a}$ without passing through $x_{h}$. We also randomly fix $x_{0}$. Figure 3 illustrates these cases. The FPT densities for these two cases are compared with the FPT density for the homogeneous case of CTRW model, when all travel time distributions are exponential with the same parameter $\tau: \tilde{\psi}_{x x^{\prime}}(s)=1 /(1+s \tau)$. We find that a random walk in HCTRW from the case (b) gives the homogeneous CTRW model, since the trap node $\mathrm{x}_{\mathrm{h}}$ cannot be reached by a random walk. At the same time, placing a trap in the same community with $\mathrm{x}_{0}$ and $\mathrm{x}_{\mathrm{a}}$ (case (a)) changes the long-time behavior of the FPT density in comparison to the homogeneous case (Fig. 3 (bottom)). In contrast, for the case (b) the long-time behavior is the same as for the homogeneous case. We note that communities structure in larger networks can be more complicated than in the scheme, Fig. 3 (top), and the HCTRW framework with placing traps in different parts of a network can be used for analysis of communities and trapping efficiency.

\section{FPT density on scale-free networks}

From our numerical results for loopless networks ("HCTRW on regular graphs" subsection) we observe that the FPT densities can differ from each other in the short-time regime. Here we numerically calculate the FPT density for the HCTRW separately in two cases of SF networks: for SF with locally tree-like structure $(m=1$ parameter of SF model) and with loops $(m>1)$. 


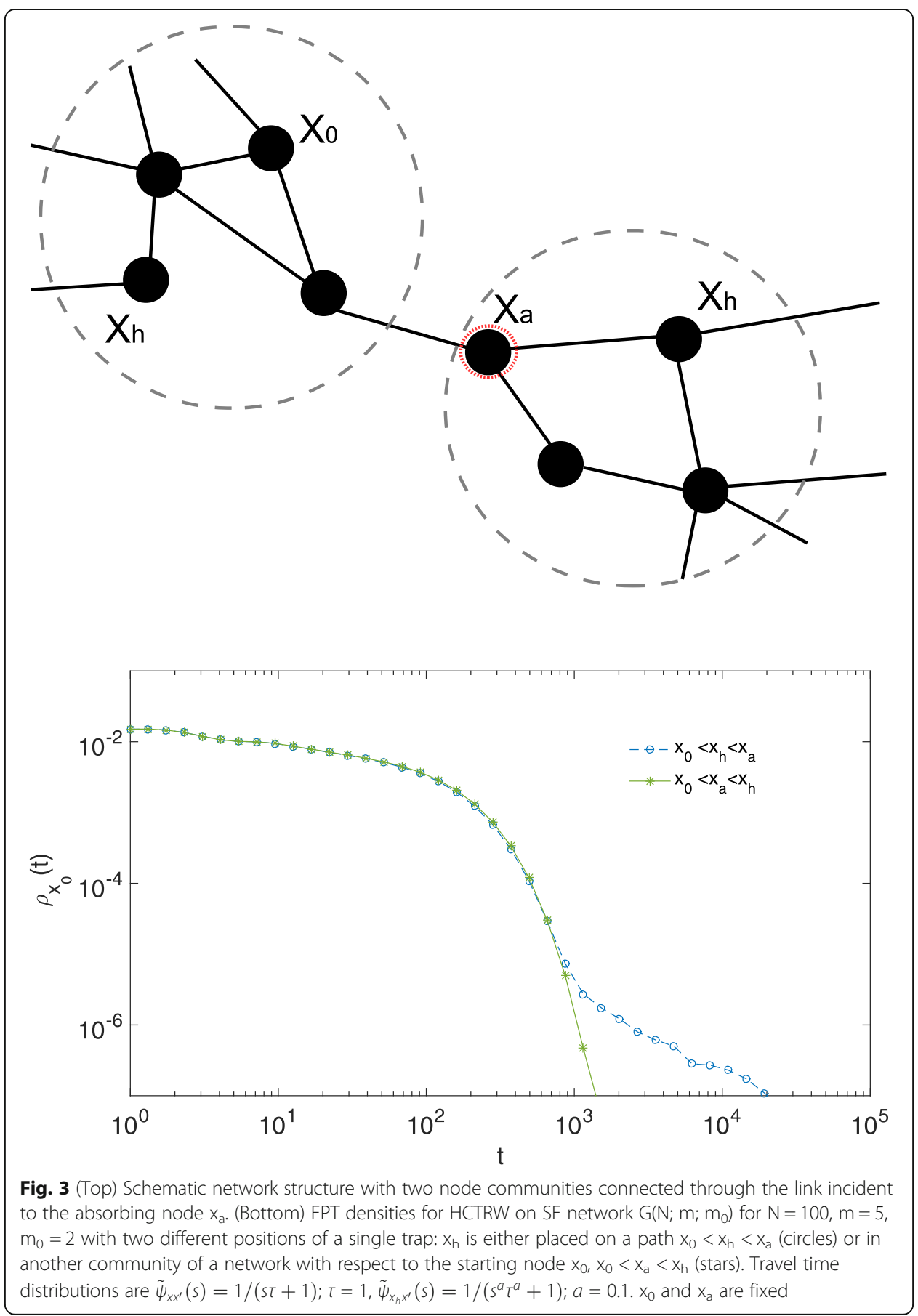

First we test the influence of the global topological properties on the FPT density and plot it for SF networks with fixed $\mathrm{m}_{0}$ and various $\mathrm{m}$ parameters and homogeneous exponential travel times. Figure 4 shows that the case $m=1$ affects mostly the short-time and intermediate-time regimes, while cases with $m>1$ slightly differ in the short-time regimes.

We fix all travel times to be independent identical exponentially distributed $\tilde{\psi}_{x x^{\prime}}(s)=1$ $/(s \tau+1) ; \tau=1$, except in $x_{h}$, at which $\tilde{\psi}_{\mathrm{x}_{\mathrm{h}} \mathrm{x}^{\prime}}(\mathrm{s})=1 /\left(\mathrm{s}^{a} \tau^{a}+1\right)^{v}, \alpha \in(0,1), v=2$, where an auxiliary parameter $v$ allows one to scatter more $\rho_{x_{0}}(t)$ for different $x_{0}$. The FPT densities 


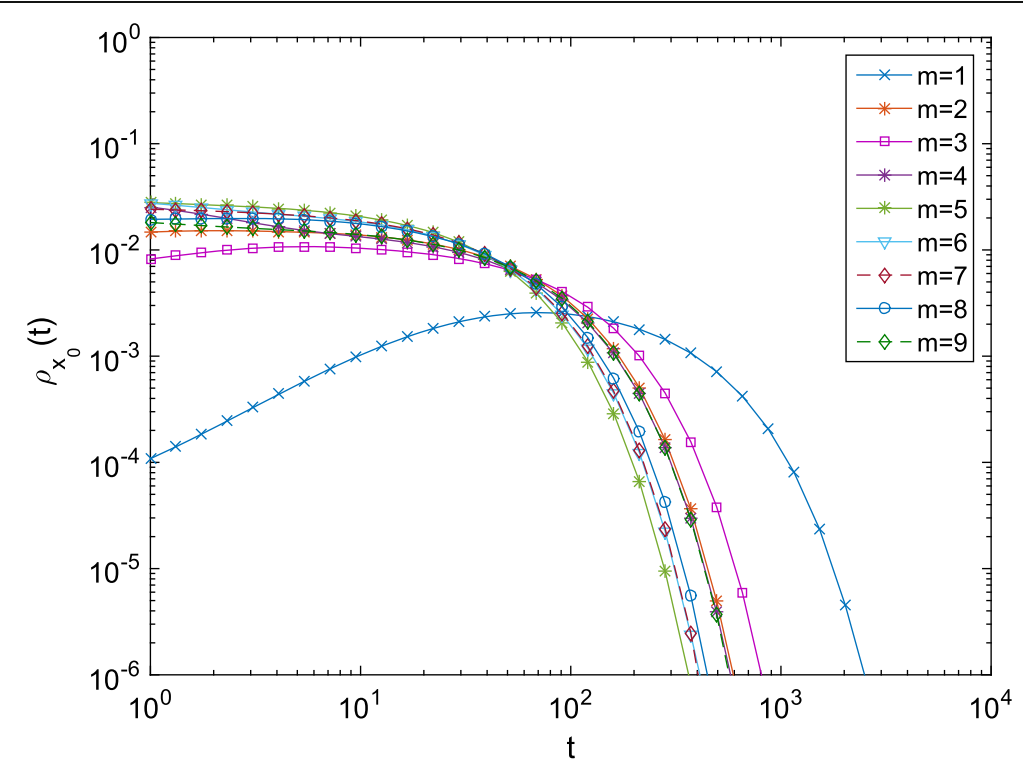

Fig. 4 FPT density for SF networks with parameters $m_{0}=11$ and $m=1$ (tree SF network), $m \in[2,9]$ (non-tree SF network) with $N=100$ nodes. Travel time distribution for all the nodes is $\tilde{\psi}_{x x^{\prime}}(s)=1 /(s \tau+1)$; $\tau=1$. The starting point $x_{0}$ and absorbing point $x_{a}$ are randomly chosen in each SF network

are computed for different starting points $x_{0}$ and fixed $x_{a}$ on a SF network (Figs. 5 and 6). Knowing that there are different shortest path distances between $\mathrm{x}_{0}$ and $\mathrm{x}_{\mathrm{a}}$, we estimate numerically the lengths of all possible shortest lengths $\left|x_{o}, x_{a}\right|$ for fixed $x_{a}$ using Dijkstra's algorithm. We numerically explore the relation between the short-time regime of the FPT density and the intrinsic network metrics, defined by the shortest paths between nodes.

For SF network with loops there exist several shortest paths between randomly taken nodes. For example, in a SF network with $m=5 ; m_{0}=6 ; N=100$, there are three groups of the shortest paths between all pairs of nodes with three different lengths. Correspondingly, we clearly observe three different early time behaviors for the FPT density, while for large time the FPT densities behave similarly for different $\mathrm{x}_{0}$. This illustrates the fact that the long-time behavior of the FPT density does not capture some important information about the system.

Next, we compare the FPT densities of SF tree and non-tree networks for different starting positions $\mathrm{x}_{0}$ (Figs. 5 and 6). We observe that changing $\mathrm{x}_{0}$ in a SF tree (Fig. 5) generally affects the whole FPT density, while changing $x_{0}$ in a SF network with $m>1$ (we show it for $m=5$ ) only affects the short-time regime. Generally, we observed that the short-time regime is affected by the geometric network properties, such as the distance between $x_{0}$ and $x_{a}$. This illustrates the fragility of the transport properties on tree-like structures. One of simple explanations is that for the same number of nodes $\mathrm{N}$ a tree network has larger variety of distances between nodes than in the case of a network with loops.

\section{FPT density on Watts-Strogatz networks}

Another example of a network with small-world property is the WS network model (Watts and Strogatz 1998). The average shortest path length M in the WS model gives 

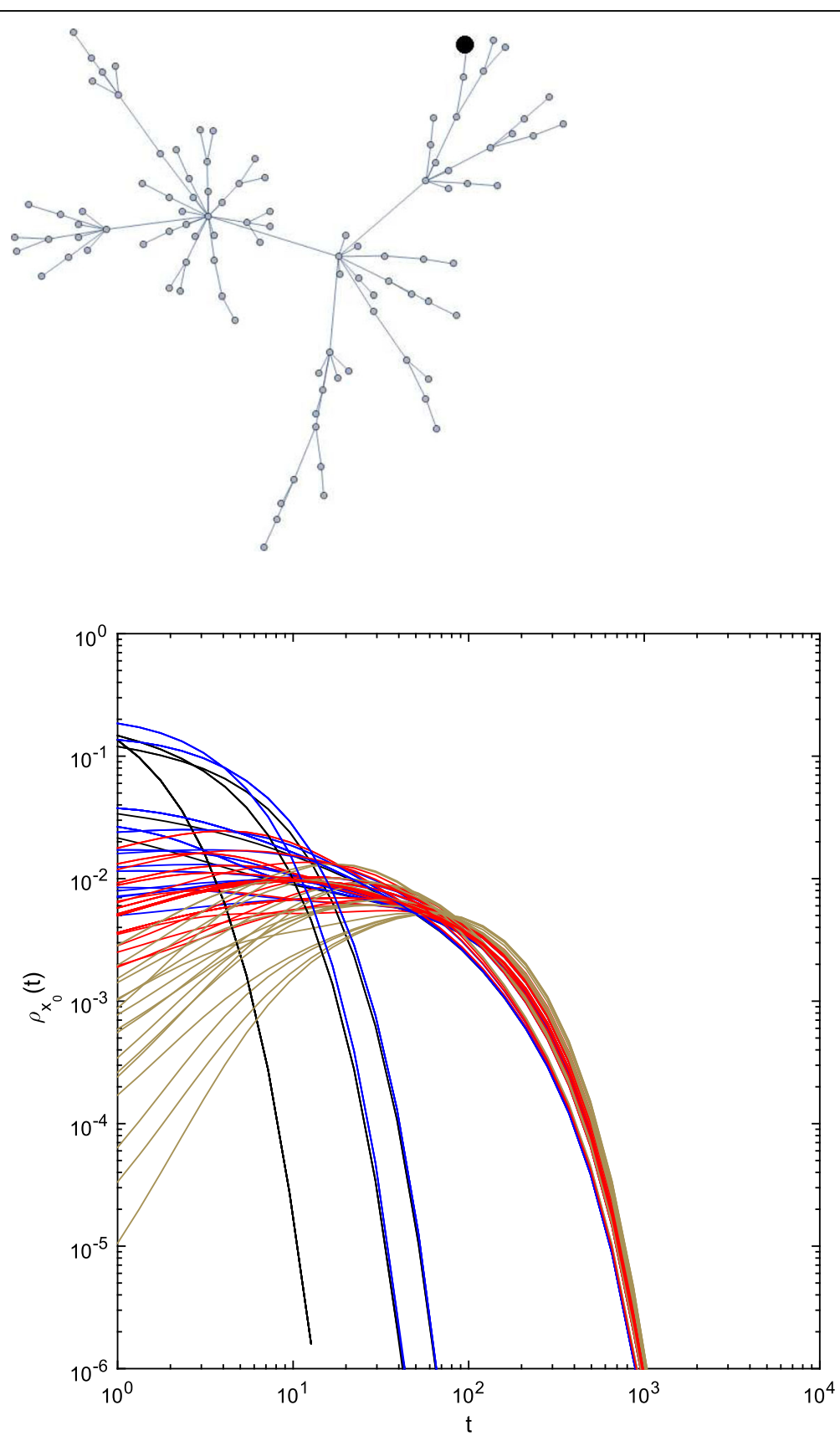

Fig. 5 (Top) Example of a SF network with the fixed absorbing node $x_{a}$ marked by black circle. (Bottom) FPT densities on $\mathrm{SF}$ network $\mathrm{G}\left(\mathrm{N} ; \mathrm{m} ; \mathrm{m}_{0}\right) \mathrm{N}=100, \mathrm{~m}=1, \mathrm{~m}_{0}=6$ for different starting points $\mathrm{x}_{0}$ and a fixed absorbing node $x_{a}$ such that: $\left|x_{0}-x_{a}\right|=1$ (black), $\left|x_{0}-x_{a}\right|=2$ (blue), $\left|x_{0}-x_{a}\right|=3$ (red) (FPT densities with $\left|x_{0}-x_{a}\right|>3$ are shown in grey). The travel time distribution is $\tilde{\psi}_{x x^{\prime}}(s)=1 /\left(s^{a} \tau^{a}+1\right)^{2} ; a=1, \tau=1$, except in a fixed trap node at which $a=0.5$

an estimate of the small-world property. For $\beta=0$ (k-circular graph) the average shortest path length is $N=4 \mathrm{k}$ (Barrat and Weigt 2000; Newman et al. 2000). While for $\beta \neq 0$ the average shortest path length is smaller than $N=4 k$. For $\beta=0.2$; $k=8$ we get $M \approx 4$, Fig. 7 (top). The FPT density for WS network with various initial positions $x_{0}$ and fixed 

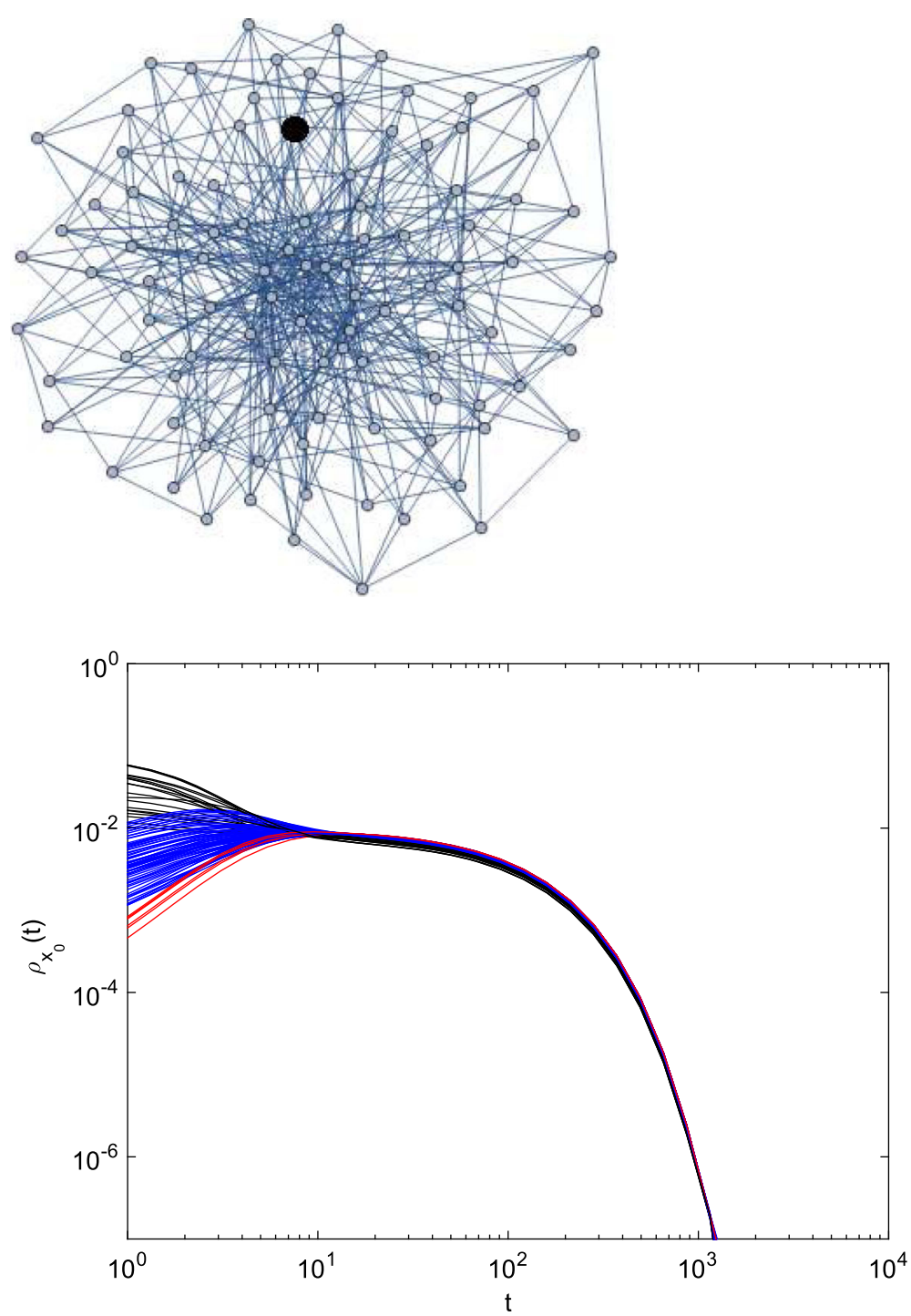

Fig. 6 (Top) Example of a SF network with the fixed absorbing $x_{a}$ marked by black circle. (Bottom) FPT densities on SF network $G\left(N ; m_{0} ; m_{0}\right) N=100, m=5, m_{0}=6$ for different starting points $x_{0}$ and a fixed absorbing node $x_{a}:\left|x_{0}-x_{a}\right|=1$ (black), $\left|x_{0}-x_{a}\right|=2$ (blue), $\left|x_{0}-x_{a}\right|=3$ (red). The travel time distribution is $\tilde{\psi}_{x x^{\prime}}(s)=1 /\left(s^{a} \tau^{a}+1\right)^{2} ; a=1, \tau=1$; except in a fixed trap node, at which $a=0.5$

$x_{a}$ are shown in Fig. 7 (bottom). Here we consider the WS network with the rewiring probability $\beta=0.2$, the average degree $k=8$ and $N=100$ nodes.

In the short-time regime we clearly see $\mathrm{m}$ distinct groups, where $m$ is the number of different shortest paths lengths in a network. Another observation is absence of a plateau in the intermediate regime (Fig. 7) for SF -networks with $m=5$ for $t$ $\in\left[10^{1} ; 10^{3}\right]$, see Fig. 6 .

\section{Analytical insights onto the FPT density}

Here we discuss the analytical calculations for the short-time and long-time regimes of the FPT density for a particular case of HCTRW model. Let us consider a path of a random walk from $\mathrm{x}_{0}$ to an absorbing node $\mathrm{x}_{\mathrm{a}}$ on a network with transition matrix $\mathrm{Q}$. 

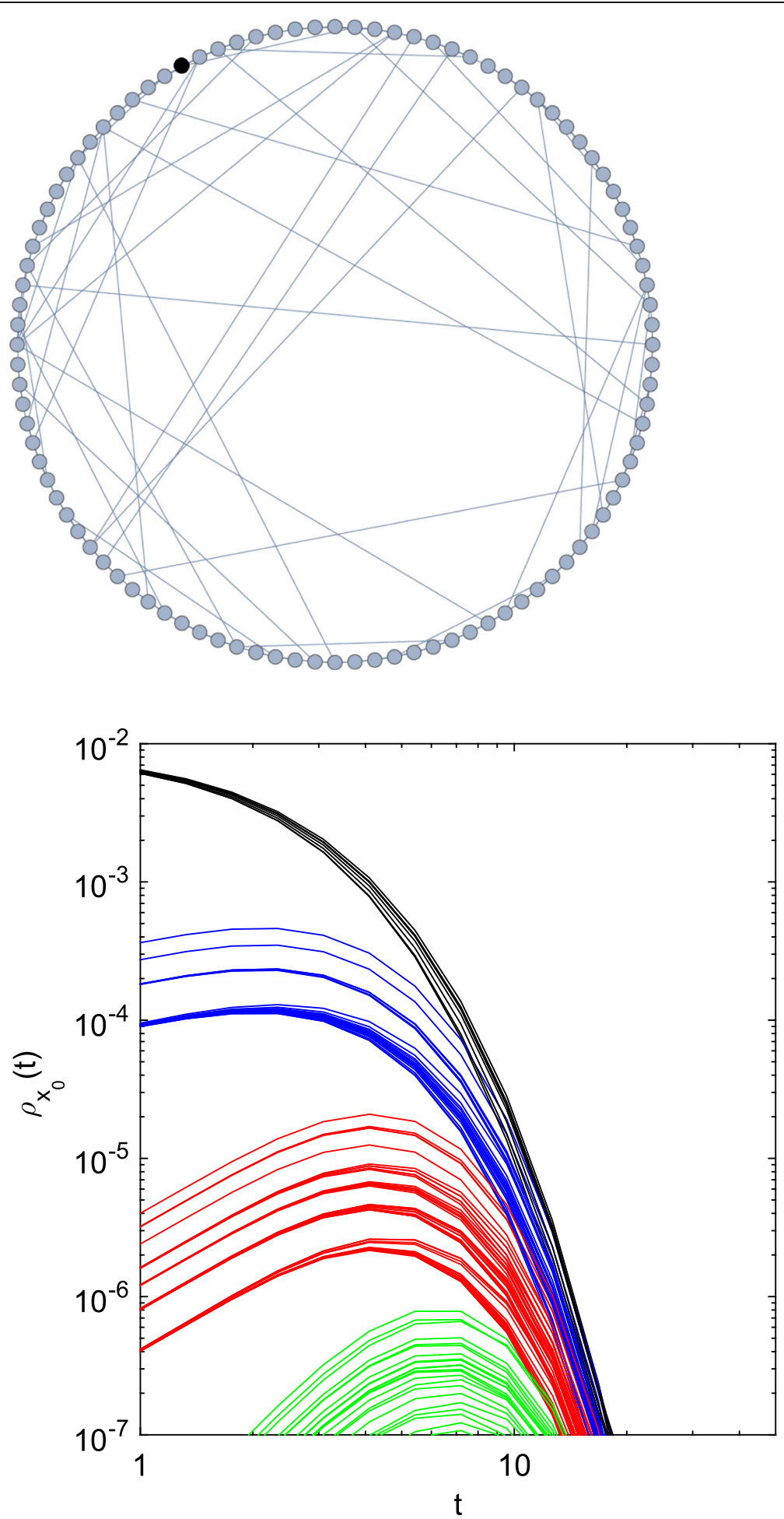

Fig. 7 (Top) Example of a WS network model with $\beta=0.2 ; k=8 ; N=100$, with a fixed absorbing node $x_{a}$ marked by black circle. (Bottom) FPT densities for this network with homogeneous travel time distributions $\tilde{\psi}_{x x^{\prime}}(s)=1 /(s \tau+1) ; \tau=1$; for different starting points $x_{0}$ and a fixed absorbing node $x_{a}=1$. Different colors correspond to different shortest paths length between $x_{0}$ and $x_{a}:\left|x_{0}-x_{a}\right|=1$ (black), $\left|x_{0}-x_{a}\right|=2$ (blue), $\left|x_{0}-x_{a}\right|=3$ (red), $\left|x_{0}-x_{a}\right|=4$ (green) 
If all travel time distributions are exponentials with the mean $(t)$, then the probability of making the path of length $n$ is:

$$
p_{n}(t)=\frac{t^{n-1}}{\tau^{n} \Gamma(n)} e^{-t / \tau}
$$

In the short-time regime the function $t^{n-1}$ dominates, while in the long-time regime the influence of the exponential $e^{-t / \tau}$ is much more profound. The total probability density of arriving at $x_{a}$ from $x_{0}$ at time $t$ is:

$$
P_{x_{0} x_{a}}(t)=\sum_{n=1}^{\infty}\left(Q^{n}\right)_{x_{0} x_{a}} p_{n}(t)=\frac{e^{-t / \tau}}{\tau} \sum_{n=1}^{\infty}\left(Q^{n}\right)_{x_{0} x_{a}} \frac{(t / \tau)^{n-1}}{(n-1) !} .
$$

The calculations of the propagator for a tree-like network can be simplified using the property that the $\mathrm{n}^{\text {th }}$ matrix power in the infinite series becomes zero matrix for $\mathrm{n} \geq \mathrm{d}$, where $\mathrm{d}$ is the diameter of a network (Petit et al. 2018). Variations of the shortest path $\left|x_{0}-x_{a}\right|$ between the starting point $x_{0}$ and the fixed absorbing node $x_{\mathrm{a}}$ affects $\left(Q^{n}\right)_{x_{0} x_{a}}$.

The long-time behavior in finite graphs, regardless of a network topology, has generic exponential tail (Bollt and ben-Avraham 2005). However, this result is valid for the long-term behavior on structures without traps. Let us consider a HCTRW model with induced structural or temporal perturbations, in which all nodes have a finite-moment distribution $\psi(\mathrm{t})$ except one node $\mathrm{x}_{\mathrm{h}}$ with a heavy-tail distribution $\psi_{x_{h}}(t)$. Then the spectral properties of the generalized transition matrix $\mathrm{Q}(\mathrm{t})$ are affected by structural $(Q \rightarrow$ $Q_{\text {pert }}$ ) and distributional ( $\psi(\mathrm{t})$ for $x_{h} \rightarrow \psi_{x_{h}}(t)$ ) perturbations (Grebenkov and Tupikina 2018). In the case of distributional perturbations the graph is kept fixed with the same transition matrix $Q$ but the travel time distributions $\psi_{x x^{\prime}}(t)$ are edge dependent and the generalized transition matrix $\mathrm{Q}(\mathrm{t})$ changes. In the case of structural perturbations, adding or removing links affects the matrix $\mathrm{Q}$ itself, as the result, the stationary distribution changes. Both structural and distributional perturbations can be analyzed from the perspectives of temporal networks. In particular, one can consider the behavior of a random walk in the HCTRW model as a simple random walk moving on continuously changing temporal network. In this temporal networks links are available in a certain time intervals, distributed according to some probability distribution $e_{x x^{\prime}}(t)$ for an edge $e_{x x^{\prime}}$. Here we refer to the frameworks presented in (Ahmad et al. 2018; Lambiotte et al. 2013; Valdano et al. 2018), where continuously evolving networks were presented.

\section{Applications to real-world networks}

Let us give an illustrative example of the HCTRW applications. If a train station with a small number of connections was shut down due to technical problems, it would not significantly increase the number of additional trips that passengers would have to take to arrive at their final destinations. However, if a random perturbation affects a station with many connections or a connecting point between several clusters of stations, the average path length can increase dramatically. In real transportation systems the road occupancy is varying over time, as such, the time of occupancy between two places $\mathrm{x}$ and $x$ ' can be modeled by a random variable distributed with $\psi_{x x^{\prime}}(t)$. Then the perturbation in the transportation liquidity (bursts) at some node can be modeled in the HCTRW as the trap $\mathrm{x}_{\mathrm{h}}$ with heavy-tail travel times. Links avoiding a trap in HCTRW model correspond to additional 


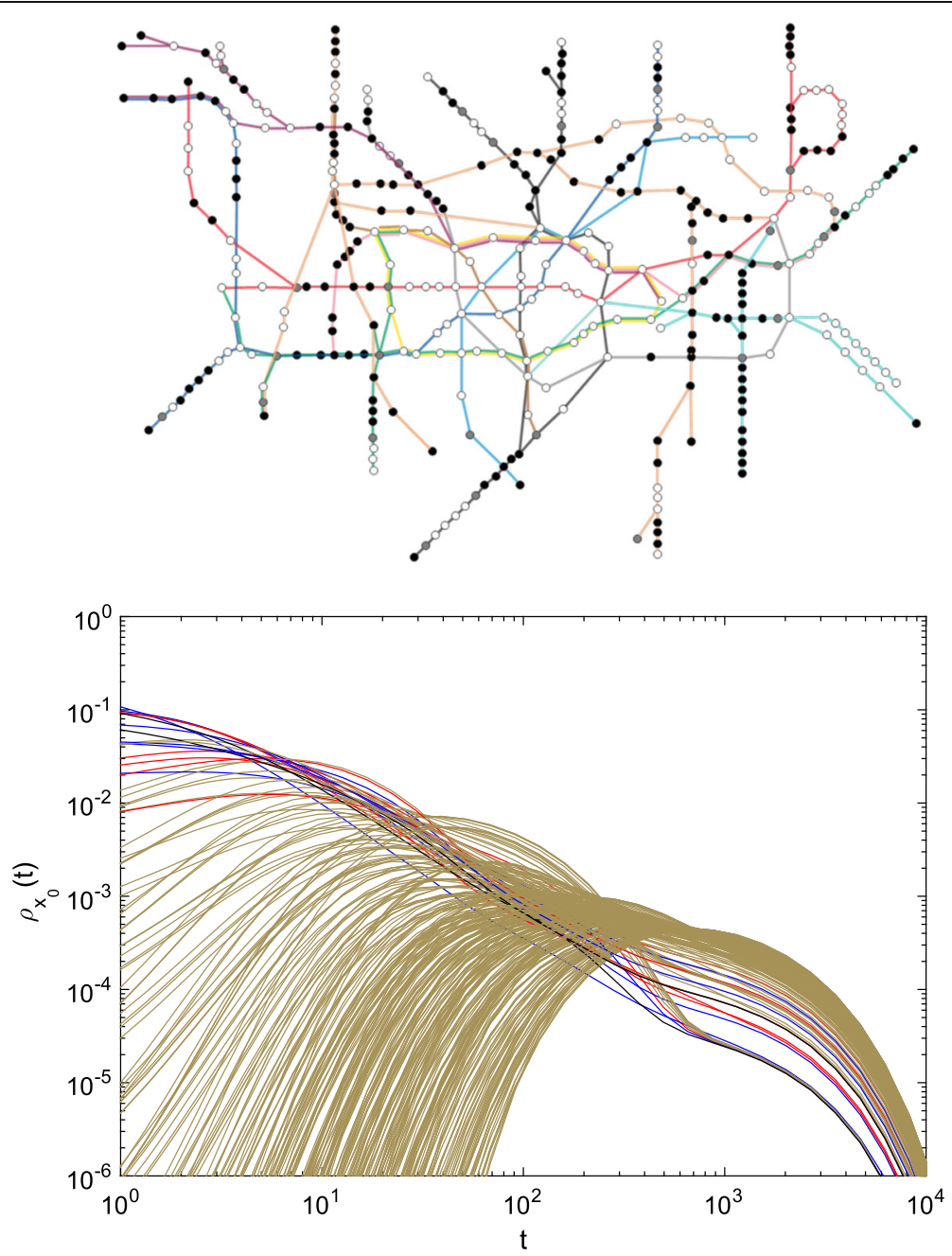

Fig. 8 (Top) The London metro map with destination Piccadilly station $x_{a}$ highlighted in black. (Bottom) FPT densities for the London metro with travel time distributions $\tilde{\psi}_{x x^{\prime}}(s)=1 /(s \tau+1) ; \tau=1$ except at a trap node $x_{h}$ at which $\tilde{\psi}_{x_{h} x^{\prime}}(s)=1 /\left(s^{a} \tau^{a}+1\right) ; a=0.5$. For each starting stations $x_{0}$ the FPT density is colored according to the distance from $x_{0}$ to $x_{\mathrm{a}}:\left|x_{0}-x_{a}\right|=1$ (black), $\left|x_{0}-x_{a}\right|=2$ (blue), $\left|x_{0}-x_{a}\right|=3$ (red) (FPT densities with $\left|x_{0}-x_{a}\right|>3$ are shown in grey)

transport connections in the real-world network, e.g. connection with buses etc. We consider a spreading entity (infection, rumour, flux of passengers, etc.), which can be modelled by a random walk behavior, although in the case of epidemics spreading the spreading quantity is not necessarily conserved. We choose to analyze the London metro (Wolfram mathematica database 2016), which shares some common features with other metro systems in the world, such as the average node degree of around 2.5 inside the core of metro network (Roth et al. 2012). The London metro on Fig. 8 (top) has $N=299$ stations (nowadays it has around 353 stations and nearly 400 edges). The core of the London metro network, neglecting the radial stations outside the circle, exhibits the small-world property. We introduce a trap (perturbation) to the London metro to see how this affects the transportation properties on the whole network. In Fig. 8 we plot the FPT densities starting from different stations of the London metro and finishing at Piccadilly circus metro station. Comparing Fig. 8 (bottom) with the FPT density for SF and WS networks (Figs. 5, 6 and 7) 
helps to get us new insights. First, the whole metro network of London is separated into several groups of nodes according to the destination, in each group the short-time regimes of $\rho_{x_{0}}(t)$ are similar. Second, the long time regime is independent from the starting point (Fig. 8).

\section{Discussions}

The properties of random walks on various complex networks have been studied extensively (Klafter and Sokolov 2011; Krapivsky et al. 2013; Steinbock et al. 2017). But quite often the asymptotic analysis and the averaged characteristics of diffusive transport and random walk dynamics, such as mean return time and mean first-passage time, are calculated (Bollt and ben-Avraham 2005; Maier and Brockmann 2017). In this manuscript we focused on studying the probability distribution of the first-passage time for random walk on complex networks with structural and temporal heterogeneities, corresponding to traps in the HCTRW model.

We identifed three regimes of the FPT density (Fig. 9): short-time, intermediate time and long-time regimes. The scheme also demonstrates how the FPT density is affected by different types of perturbations. In particular, local structural perturbations (such as an inclusion of links avoiding $x_{h}$ ) mainly affect the short-time regime of the FPT density. The global structural perturbations are the accumulated local perturbations of the network structure, e.g. the perturbations, which cannot be described by adding or removing links between already incident nodes. These perturbations seem to effect the most the intermediate regime of FPT density. Finally, the distributional perturbations (when the travel times $\psi_{x x^{\prime}}(t)$ are link-dependent) are mainly affecting the long-time regimes of the FPT density. One can also consider the interplay of different types of perturbations but this goes beyond the scope of this paper. We start with the least studied short-time regime. We emphasize that the comparison of the first-passage times for different types of networks is qualitative. We draw the

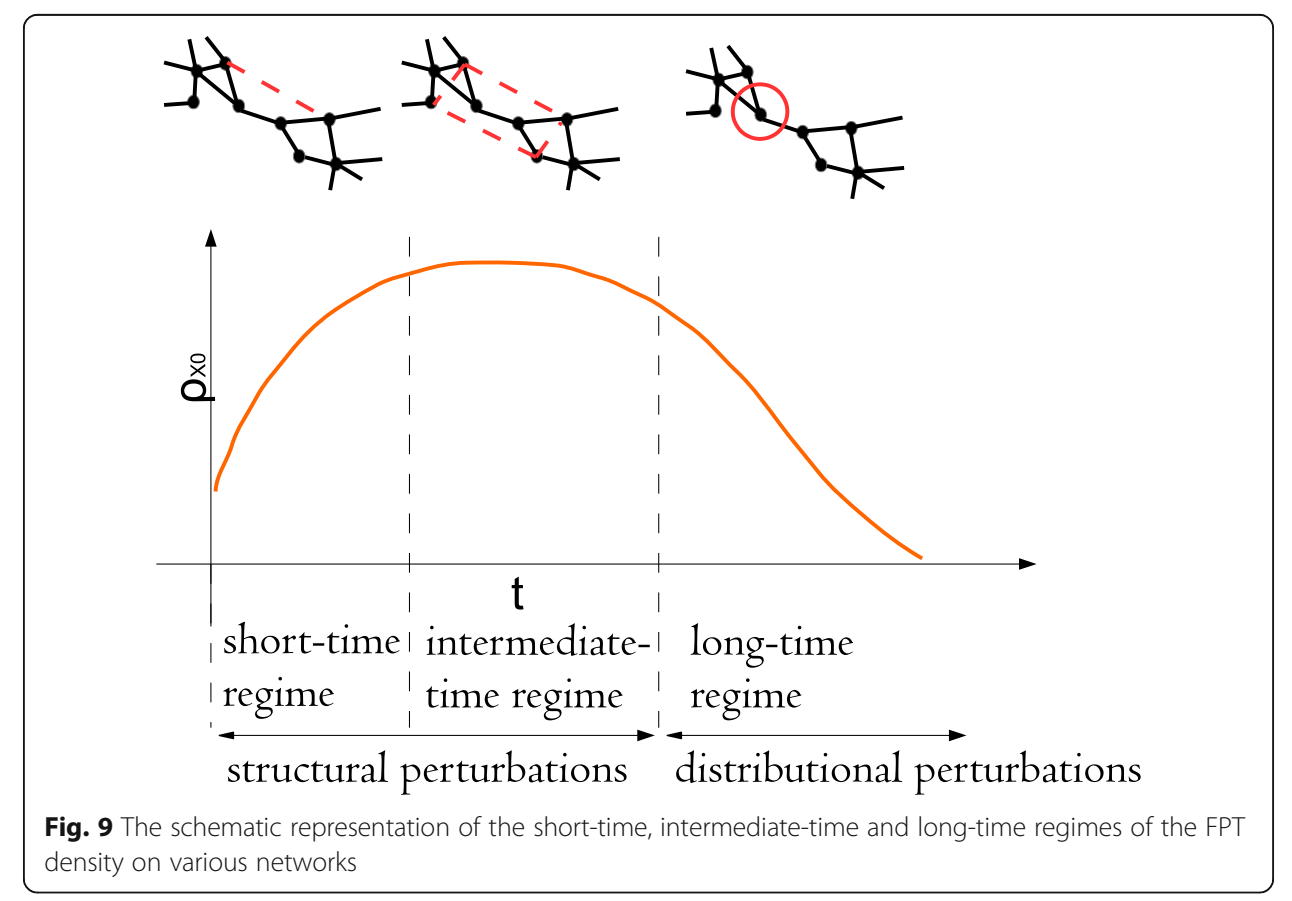


separate conclusions for each type of network, from which we highlight common observations.

The short-time regime of the first-passage time is generally affected by the geometric network properties, such as the distance between $\mathrm{x}_{0}$ and $\mathrm{x}_{\mathrm{a}}$, which can be calculated, as the minimal power of the adjacency matrix of a network with nonzero element $\mathrm{A}_{x_{0} x_{a}}:\left(\mathrm{A}^{\mathrm{L}-1}\right)_{x_{0} x_{a}}=0$; $\left(\mathrm{A}^{\mathrm{L}}\right)_{x_{0} x_{a}} \neq 0$ : As we saw from the numerical computations of $\rho_{x_{0}}(t)$ ("HCTRW on regular graphs" subsection), a local perturbation induced by an avoiding link strongly affects the short-time regime (Fig. 2 (bottom)). In other words, local changes of a network structure affect the distribution of the shortest paths, which in turn affects the left tail of the FPT density (Figs. 5 and 6). In the same vein, the FPT properties are strongly affected by the metrics of a graph and not just by the node degrees; on the other hand, node importance and general transport properties of networks do not depend exclusively on node degrees (Kitsak et al. 2010). At the same time, the non-linear network measure distribution is related to dynamical properties in a rather complex way and should be analyzed separately.

The intermediate-time regime of the FPT density mainly depends on the global topological features of a network (i.e. small-world property, loopless structure). For some networks the intermediate regime is less pronounced than for others (compare Figs. 5 and 6). For instance, for WS model (Fig. 7), the intermediate regime between the most probable first-passage time, $t_{\mathrm{mp}}$, and beginning of a decrease of the FPT density is not so well pronounced. For networks without traps changing the shortest path between $x_{0}$ and $x_{a}$ shifts the maximum of the FPT. This resembles the properties of diffusion in continuous domains (Godec and Metzler 2016), where $t_{\mathrm{mp}}$ is proportional to the squared distance between the starting point and the target. Another important characteristics of the intermediate regime is the presence of a plateau, like the intermediate regime at $t \in\left[10^{1} ; 10^{3}\right]$ for SF networks with $\mathrm{m}=5 ; \mathrm{m}_{0}=6$ (Fig. 6), which is a general feature present also for FPT in continuous domains (Grebenkov et al. 2018).

Finally, the long-time regime is largely influenced by temporal heterogeneities, i.e. the trap node $x_{h}$ with a heavy tail distribution of travel time $\psi_{x_{h} x^{\prime}}(t)$. In particular, it was identified that for simple random walks, the MFPT (mean first-passage time) captures some long-time properties of the dynamics. However, MFPT neglects some important information about the process, significantly overestimating the scales of the most-probable first-passage time (Godec and Metzler 2016; Grebenkov et al. 2018). At the same time it is known that for dynamics of a particle diffusing in a continuous domain the MFPT is of order $\mathrm{L}^{2} / \mathrm{D}$, where $\mathrm{L}$ is the domain size, $\mathrm{D}$ is the diffusion coefficient (Grebenkov et al. 2018; Singer et al. 2006). In the continuous case the most probable FPT is shown to strongly depend on the starting position and is almost independent of the target properties (Grebenkov et al. 2018). How the dynamical properties are changing when a distributional perturbation at $x_{h}$ is introduced? The influence of the trap $x_{h}$ on the long-time regime is illustrated on Fig. 3, where placing $x_{h}$ in different communities of a network changes the right tail of the FPT density. Note that a trap does not affect a geometric length of a path between $x_{0}$ and $x_{a}$, but may affect the shortest-in-time path between nodes. Hence even if there are several paths between nodes there is a non-zero probability for a random walk to reach $\mathrm{x}_{\mathrm{h}}$ at long times, which then may affect the right tail of the distribution (Fig. 1). To complete our discussions on this point, we recall that the properties in the long-time regime of random walks on networks without heterogeneities have been proven to depend on the dimension 
of a random walk (O'Shaugnessy and Procaccia 1985). Moreover, in the case of the compact random walk exploration, e.g. HCTRW on Vicsek fractals with spectral dimension $d_{s}<2$ (Fig. 2), the asymptotic properties of the FPT density do not depend on a degree of a target node and have the power law decay $t^{-2 d_{s}-1-a}$.

\section{Conclusions}

In this article we considered the HCTRW framework for the analysis of the feature-rich networks, in particular, we studied effects of heterogeneity on random walk dynamics in several types of networks. We explored FPT densities on regular and irregular structures, such as regular fractals, random Scale-Free and Watts-Strogatz network models. The comparison of regular versus irregular structures allowed us to refine some properties of the FPT densities on complex networks. Notably, we analyzed effects of structural and distributional heterogeneities using the first-passage time as one of the key indicators of how fast information diffuses in a given system (Maier and Brockmann 2017; Redner 2001). Heterogeneities are encoded in the generalized transition matrix Q(t) (Grebenkov and Tupikina 2018), which affects its spectral properties and as the result the dynamical properties of processes on such networks. The influence of the structural and distributional perturbations of network structure on FPT density is summarized in Fig. 9. The local and global structural perturbations mainly influence the short-time and intermediate-time regimes. The distributional perturbations generally affect the long-time regime. We find that not only the topology of a network but also temporal discrepancy encoded in the distributional heterogeneities $\psi_{x x^{\prime}}(t)$ can significantly alter the behavior of random walk on a network.

In particular, we found that the short-time regime depends on the distribution of distances between starting and absorbing nodes in a network. The existence of loops generally influences the dynamical properties of random walks on these graphs (Dorogovtsev and Mendes 2002). For the HCTRW model the changes of global network structure infuence the intermediate-time regime of the FPT density, e.g. SF network with or without cycles has different intermediate regime duration ("Results" section). In general, analysis of the short-time and intermediate-time regimes allows us to characterize transport properties of networks even when the first moment of the distribution is infinite (Hernandez-Garcia and Caceres 1990).

To summarize, the FPT density provides us with alternative characteristics of network topology in addition to known static measures, such as degree or closeness network measures (Barthelemy 2011). The average number of distinct sites, visited by HCTRW and other observables derived from the FPT densities can be a quantity with the practical relevance for characterization of spreading processes.

The presented analysis and HCTRW framework dynamics can be further explored on real-world networks. The CTRW framework was used to analyze human travel laws in the continuous domains (Brockmann et al. 2006) and the HCTRW model is a compelling extension of such analysis. We applied the HCTRW model on a network graph of the London metro. The next step would be to compare the results from HCTRW simulations (Fig. 8), with the original data of traveling passengers on real-world networks (Open Data Stanford platform n.d.). Another possible application of the HCTRW framework is to dissect complex networks topologies via introducing the structural and 
temporal heterogeneities in the HCTRW model. Since the heterogeneities can be introduced to the particular nodes, this can help to verify the importance of the node to the transport. After almost a decade of scientific research, there is no definitive method of characterization of communities structures in networks, in particularly, for feature-rich networks (Barthelemy 2011; Schaub et al. 2017). Therefore the frameworks to study heterogeneous network models, such as the HCTRW framework, can add the valuable contribution in the future research of network science.

Transportation networks and the information transmission systems are the network examples, to which the HCTRW framework can be further applied, taking into account their nontrivial stochastic nature (Barthelemy 2011; Larson 2017). Moreover, the HCTRW framework can be used in order to navigate in the network using detection of the relevant search strategies, e.g. placing heterogeneities in different parts of network. Using the first-passage time observables one can further design network measures in order to characterize transport efficiency and spreading. The straight-forward way to characterize the transport and search efficiency is to use the concept of survivability as a measure of reachability of some quantity in a network, which we plan to explore in the future. We expect the framework presented in this paper to broaden the scopes of exploration of the feature-rich networks.

\section{Abbreviations}

FPT density: First-passage time density; HCTRW model: Heterogeneous Continuous Time Random Walk model; SF network: Scale-free network; WS network: Watts-Strogatz network

\section{Acknowledgements}

L.T. thanks M. Dolgushev for exchange of materials about fractal networks and P. Holme for discussions about network measures for spreading. We thank reviewers for useful and interesting comments.

\section{Funding}

The authors acknowledge the support under Grant No. ANR-13-JSV5-0006-01 of the French National Research Agency. L.T. acknowledges support of Centre of Research and Interdisciplinarity, Paris Descartes University, France.

Availability of data and materials

The datasets used and/or analyzed during the current study are available from the corresponding author on reasonable request.

\section{Authors' contributions}

LT performed numerical simulations, analyzed and interpreted the analytical and numerical results of the HCTRW model on various types of networks. DG analyzed and interpreted the analytical and numerical results. Both authors contributed in writing the manuscript. Both authors read and approved the final manuscript.

\section{Competing interests}

The authors declare that they have no competing interests.

\section{Publisher's Note}

Springer Nature remains neutral with regard to jurisdictional claims in published maps and institutional affiliations.

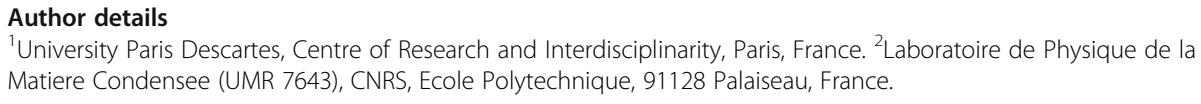


Blumen A, von Ferber C, Jurjiu A, Koslowski T (2004) Generalized Vicsek fractals: regular hyperbranched polymers.

Macromolecules 37:638-650

Bollt EM, ben-Avraham D (2005) What is special about diffusion on scale-free nets? New J Phys 7:26

Brockmann D, Hufnage L, Geisel T (2006) The scaling laws of human travel. Nature 439:462-465

Chung F (1994) Spectral graph theory. Am Math Soc 92:0160-7642

Cohen R, Erez K, Ben-Avraham D, Havlin S (2000) Phys Rev Lett 85:4626

Dorogovtsev S, Mendes JFF (2002) Evolution of networks. Adv Phys 51:1079-1187

Godec A, Metzler R (2016) First passage time distribution in heterogeneity controlled kinetics: going beyond the mean first passage time. Sci Rep 6:20349

Grabow C, Grosskinsky S, Timme M (2012) Small-world network spectra in mean-field theory. Phys Rev Lett 108:218701

Grebenkov DS, Metzler R, Oshanin G (2018) Towards a full quantitative description of single-molecule reaction kinetics in biological cells. Phys Chem Chem Phys 20:16393-16401

Grebenkov DS, Tupikina L (2018) Heterogeneous continuous-time random walks. Phys Rev E 97:012148

Havlin S, ben-Avraham D (2002) Diffusion in disordered media. Adv Phys 51:187-292

Hernandez-Garcia E, Caceres MO (1990) First-passage-time statistics in disordered media. Phys Rev A 42:8

Hudghes B (1995) Random walks in random environments. Clarendon, Oxford

Hwang S, Lee D-S, Kahng B (2012) First passage time for random walks in Heteroge-neous networks. Phys Rev Lett 109:088701

Julaiti A, Wu B, Zhang Z (2013) Eigenvalues of normalized Laplacian matrices of fractal trees and dendrimers: analytical results and applications. J Chem Phys 138:204116

Kitsak M, Gallos LK, Havlin S, Liljeros F, Muchnik L, Stanley E, Makse HA (2010) Identifying influential spreaders in complex networks. Nat Phys 6(11):888-893

Klafter J, Sokolov IM (2011) First steps in random walks. Oxford University Press

Krapivsky P, Redner S, Ben-Naim E (2013) A kinetic view of statistical physics. Cambridge University Press

Lambiotte R, Rocha LEC, Delvenne J-C (2015) Diffusion on networked systems is a question of time or structure. Nat Comm 6:7366

Lambiotte R, Tabourier L, Delvenne J-C (2013) Burstiness and spreading on temporal networks. Eur Phys J B 86:320

Larson JM (2017) The weakness of weak ties for novel information diffusion. App Net Sci 2:14

Liu H, Dolgushev M, Qi Y, Zhang Z (2015) Laplacian spectra of a class of small-world networks and their applications. Sci Rep 5:9024 Maier B, Brockmann D (2017) Cover time for random walks on arbitrary complex networks. Phys Rev E 96:042307

Mieghem PV (2011) Graph spectra for complex networks. Cambridge University Press

Monti C, Boldi P (2017) Estimating latent feature-feature interactions in large feature-rich graphs. Internet Math J 10:24166

Montroll E, Weiss G (1965) J Math Phys 6:167

Newman ME, Moore C, Watts DJ (2000) Mean- field solution of the small-world network model. Phys Rev Lett 84:32013204 Open Data Stanford platform snap.stanford.edu/data/

O'Shaugnessy B, Procaccia I (1985) Analytical solutions for diffusion on fractal objects. Phys Rev Lett 54:455

Petit J, Gueuning M, Carletti T, Lauwens B, Lambiotte R (2018) Random walk on temporal networks with lasting edges, arxiv 1809.02540

Redner S (2001) A guide to first-passage processes. Cambridge University Press

Roth C, Kang SM, Batty M, Barthelemy M (2012) Evolution of subway networks. J R Soc Interface 9:2540-2550

Schaub MT, Delvenne J-C, Rosvall M, Lambiotte R (2017) The many facets of community detection in complex networks. Appl Netw Sci 2:4

Singer A, Schuss Z, Holcman D (2006) Narrow escape, part II: the circular disk. J Stat Phys 122:465-489

Sood V, Redner S (2005) First-passage properties of the Erdos-Renyi random graph. J Phys A 38:109123

Steinbock C, Biham O, Katsav E (2017) Distribution of shortest path lengths in a class of node duplication network models. Phys Rev E 96(3):032301

Talbot A (1979) The accurate numerical inversion of Laplace transforms. J Inst Maths Appl 23:97-120

Valdano E, Re Fiorentin M, Poletto C, Colizza V (2018) Epidemic threshold in continuous-time evolving networks. Phys Rev Lett 120:068302

von der Hofstadt R (2017) Random graphs and complex networks. Cambridge University Press

Watts DJ, Strogatz SH (1998) Collective dynamics of 'small-world' networks. Nature 393:440-442

Wolfram mathematica database, London tube data (2016)

\section{Submit your manuscript to a SpringerOpen ${ }^{\circ}$ journal and benefit from:}

- Convenient online submission

- Rigorous peer review

- Open access: articles freely available online

- High visibility within the field

- Retaining the copyright to your article

Submit your next manuscript at $\mathbf{s p r i n g e r o p e n . c o m ~}$ 